\title{
Minimally-invasive neonatal surgery: laparoscopic excision of choledochal cysts in neonates
}

\author{
Hyo Seon Ryu', Ju Yeon Lee ${ }^{2}$, Dae Yeon Kim¹, Seong Chul Kim¹, Jung-Man Namgoong' \\ ${ }^{1}$ Department of Pediatric Surgery, Asan Medical Center Children's Hospital, University of Ulsan College of Medicine, Seoul, Korea \\ ${ }^{2}$ Department of Pediatric Surgery, Chonnam National University Children's Hospital, Chonnam National University School of \\ Medicine, Gwangju, Korea
}

\begin{abstract}
Purpose: Improvements in surgical techniques and a better understanding of the unique anesthetic requirements in neonates undergoing laparoscopy have suggested that laparoscopic surgery may be effective in newborns. This study therefore evaluated the safety and feasibility of laparoscopic excision of the cyst (LEC) in neonates.

Methods: This retrospective study included 43 neonates who underwent excision of choledochal cysts between November, 2001, and January, 2018, including 21 who underwent open excision and 22 who underwent LEC. Their perioperative and surgical outcomes were reviewed. The patients were followed up for a median 37 months (range, 3-141 months).

Results: Baseline characteristics did not differ significantly in the open and LEC groups. Mean intraoperative peak partial pressure of arterial $\mathrm{CO}_{2}\left(\mathrm{PaCO}_{2}\right)(45.5 \mathrm{mmHg}$ vs. $48.0 \mathrm{mmHg})$ and total operation time (208.3 \pm 71.0 minutes vs. $235.0 \pm 47.2$ minutes) were similar in both groups. Parents of the patients in the LEC group provided a more positive evaluation of scar scale and greater satisfaction with wound. No patient in either group experienced any critical complications. Three patients in the open excision group required readmission for cholangitis and 2 patients had ileus. No patient in the laparoscopic excision group experienced any postoperative complications during follow-up.

Conclusion: Despite difficulties performing laparoscopic surgery in neonates, LEC was safe and feasible when intraperitoneal peak pressure was maintained under $10 \mathrm{mmHg}$ and $\mathrm{PaCO}_{2}$ was closely monitored by a pediatric anesthesiologist. Compared with open excision, LEC provided improved cosmetic outcomes without severe complications. Prospective randomized studies with large numbers of patients are warranted.

[Ann Surg Treat Res 2019;97(1):21-26]
\end{abstract}

Key Words: Choledochal cyst, Laparoscopy, Infant, Minimally invasive surgical procedures

\section{INTRODUCTION}

Choledochal cyst $(\mathrm{CC})$ is a congenital dilatation of the biliary tract. Because CCs can cause recurrent pancreatitis, cholangitis, sludge and gallstones, as well as having a potential for malignant changes, CCs should be surgically excised. In neonates, CCs may appear as a near total obstruction. The surgical treatment of choice consists of cyst excision and biliary bypass [1-3]. Improvements in surgical techniques and instruments have made laparoscopic excision of the cyst (LEC) and Roux-en-Y hepaticojejunostomy the preferred method in children and adults [4-7]. Laparoscopic hepaticojejunostomy in children with CC has been shown safe with lower morbidity rates than open procedures. The laparoscopic procedure provides a magnified view of the deep anatomic structure, such as the hepatic hilum, portal vein, and hepatic arteries, as well as allowing meticulous dissection and preventing injuries to critical structures. In addition, the minimally-invasive approach
Received February 11, 2019, Revised April 13, 2019,

Accepted May 2, 2019

Corresponding Author: Jung-Man Namgoong

Department of Surgery, Asan Medical Center, University of Ulsan College

of Medicine, 88 Olympic-ro 43-gil, Songpa-gu, Seoul 05505, Korea

Tel: +82-2-3010-1512, Fax: +82-2-474-6701

E-mail: namgoong2940@naver.com

ORCID code: https://orcid.org/0000-0002-9237-7440
Copyright (C) 2019, the Korean Surgical Society

(c) Annals of Surgical Treatment and Research is an Open Access Journal. All articles are distributed under the terms of the Creative Commons Attribution NonCommercial License (http://creativecommons.org/licenses/by-nc/4.0/) which permits unrestricted non-commercial use, distribution, and reproduction in any medium, provided the original work is properly cited. 
reduces rates of postoperative ileus, reduces hospital stay and provides excellent cosmetic results [8-10]. Laparoscopic surgery in neonates is not widely performed due to their sensitivity to changes in end-tidal $\mathrm{CO}_{2}$ and intraperitoneal pressure, as well as anatomic difficulties and the requirement for specialized pediatric anesthesiologists [11,12]. Recent improvements in surgical techniques and better understanding of the unique anesthetic requirements of laparoscopy in neonates have increased the ability to perform laparoscopic surgery in newborns. Fujimoto et al. [11] reported that various types of laparoscopic surgery were safely performed in 65 newborns younger than 30 days. The advantages of the laparoscopic approach in neonates with CC have not been determined. Based on our experience excising CCs in older children and our performance of other laparoscopic procedures in neonates, including in the treatment of duodenal atresia and inguinal hernias, we designed a laparoscopic method of biliary reconstruction in neonates. This study was therefore designed to evaluate the safety and feasibility of LEC in neonates.

\section{METHODS}

\section{Subjects}

The medical records of 43 consecutive neonates who underwent excision of CCs from November, 2001, to January, 2018, at the Department of Pediatric Surgery, Asan Medical Center, were reviewed retrospectively. Their perioperative outcomes and short-term morbidities were evaluated. Of these 43 neonates, 21 underwent open excision of CC (OEC), and 22 underwent LEC. Parents of all patients were surveyed through telephone questionnaires about scar scale and their satisfaction with the wound.

This retrospective study was approved by the Asan Medical Center Institutional Review Board (approval number: 20161325). Patient informed consent was waived because of the retrospective nature of the study.

\section{Operative methods}

All patients in both groups underwent standard preoperative preparation. After induction, a Foley catheter was inserted for urinary drainage and an orogastric tube was inserted for decompression of the stomach. A central line and an arterial line were inserted for hemodynamic monitoring. If the patient was stable and available, preoperative magnetic resonance cholangiopancreatography (MRCP) was performed. If not, intraoperative cholangiography via the cystic duct was performed. The dissection of pathologic CC was started from the anterior wall of the cyst. The dissection was extended inferiorly, followed by circumferential dissection and encircling. The distal common bile duct was dissected to just within the head of pancreas, then transected after double ligation.
In both groups, the proximal and distal bile duct lumens were irrigated with saline for removal of choledocholithiasis. Hepaticojejunostomy and jejunojejunostomy were performed with interrupted sutures and a retrocolic maneuver.

\section{Open excision of cyst}

The patient was placed in a supine position. An upper transverse or right subcostal incision was made. Dissection was performed to the bifurcation of the hepatic ducts, followed by circumferential transection immediately distal to the bifurcation. Stay sutures were inserted laterally and anteriorly to improve the operative field, making hepaticojejunostomy easier to perform. Reconstruction was performed with a Roux-en-Y conduit of the jejunum. The jejunum was divided with a linear stapler approximately 10 to $15-\mathrm{cm}$ downstream from the ligament of Treitz. End-toside jejunojejunostomy was performed in a single layer $>40$ $\mathrm{cm}$ below the hepaticojejunostomy. The conduit was brought through a retrocolic window. End-to-side hepaticojejunostomy was performed in a single layer. A drainage tube was positioned posterior to the hepaticojejunostomy through the foramen of Winslow and externalized through a separate incision in the right lower quadrant.

\section{Laparoscopic excision of the cyst}

LEC was performed in a manner similar to that of OEC. The patient was placed in the reverse-Trendelenburg position under general anesthesia. A supraumbilical incision was made for insertion of the 5 -mm-sized trocar and the $30^{\circ}$ laparoscope. Pneumoperitoneum was created by carbon dioxide at a pressure of 8-10 mmHg. Three additional 3-mm-sized trocars were inserted, in the right and left subcostal areas and $5 \mathrm{~cm}$ below the left subcostal port (Fig. 1). If MRCP was not performed, intraoperative cholangiography was performed through the fundus of the gallbladder. The gallbladder and falciform ligament were separately suspended through the abdominal wall with nylon sutures. The cyst was transected close to the hilum, and the remnant cyst was completely and gently excised. The distal portion of cyst was cut after tie ligation (Fig. 2). The proximal jejunum was exteriorized through an extended umbilical port incision, and a Roux-en-Y jejunojejunostomy was constructed manually with a single layer interrupted 5-0 polydioxanone suture (PDS). A retrocolic hepaticojejunostomy was constructed laparoscopically with a single layer interrupted alternating 6-0 PDS and 6-0 vicryl sutures. A drainage tube was positioned posterior to the hepaticojejunostomy through the foramen of Winslow and the right subcostal port site.

\section{Statistical analysis}

All statistical analyses were performed using IBM SPSS ver. 18.0 (IBM Co., Armonk, NY, USA). Categorical variables were 
expressed as number and frequency, and continuous variables were expressed as mean and standard deviation, or median and interquartile range (IQR). Categorical variables were compared by Fisher exact tests and continuous variables by t-tests. A P-value $<0.05$ was considered statistically significant.

\section{RESULTS}

This study included 43 neonates with CC; of these, 21 underwent OEC and 22 underwent LEC. Three patients in the OEC group were initially scheduled to undergo LEC. However, one required conversion to open surgery because of intraoperative bleeding, and the other 2 required conversion to open conversion because of a ruptured cyst with severe inflammation around the extrahepatic duct (Fig. 3).

The baseline characteristics of the patients in the 2 groups are presented in Table 1 . Seventeen of the 21 patients $(81 \%)$ in the OEC group and 19 of 22 (86\%) in the LEC group were girls. The median gestational ages of patients were 39 weeks (IQR, 38-40 weeks) in the OEC group and 38 weeks (IQR, 37-39 weeks) in the LEC group. Type I CC was dominant in both groups. Median cyst diameter on MRCP was $4.0 \mathrm{~cm}$ (IQR, 2.6-6.0 cm) in the OEC group and $3.35 \mathrm{~cm}(\mathrm{IQR}, 2.5-4.5 \mathrm{~cm})$ in LEC group. There were no significant differences between the 2 groups in preoperative laboratory parameters including total bilirubin concentrations and liver function test.

Perioperative outcomes are shown in Table 2. Of the 21 patients in OEC group, 3 (14.3\%) had ruptured cysts, 2 (9.5\%) had preoperative pancreatitis, and 2 (9.5\%) required red blood cell transfusion because of preoperative anemia. The estimated blood loss was minimal in all patients, except for one neonate who required conversion to open surgery due to intraoperative

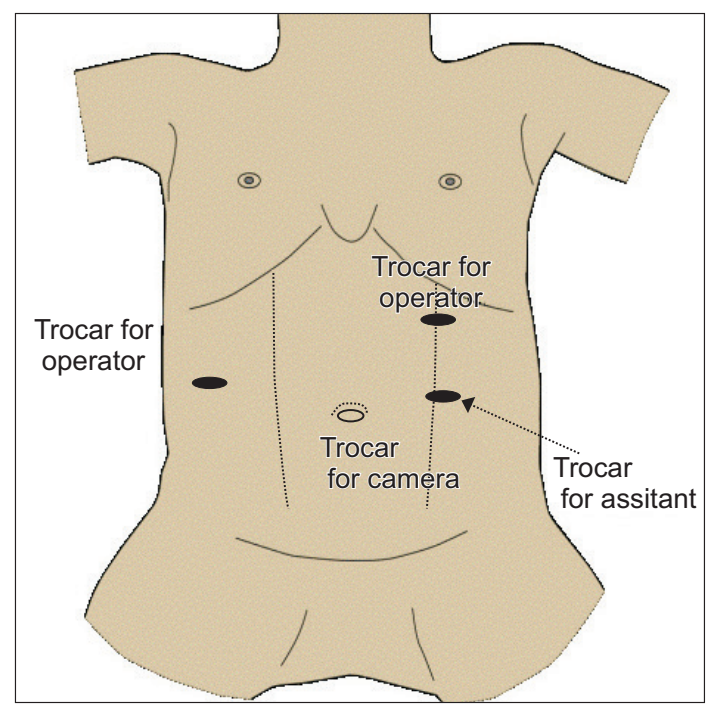

Fig. 1. Position of trocar. bleeding. In the LEC group, 2 neonates (9.1\%) had pancreatitis.

The mean intraoperative peak partial pressure of arterial $\mathrm{CO}_{2}\left(\mathrm{PaCO}_{2}\right)$ was $45.5 \mathrm{mmHg}$ in the OEC group and 48.0 $\mathrm{mmHg}$ in the LEC group ( $\mathrm{P}=0.861)$. Moreover, mean total operation times were similar in the OEC $(208.3 \pm 71.0$ minutes) and LEC (235.0 \pm 47.2 minutes) groups. There were no significant between group differences in anastomosis size, days of hospitalization, time to diet and time to bilirubin normalization.

A questionnaire was administered to the parents of all neonates assessing scar scale ( $1=$ ugliest to $10=$ invisible) and satisfaction with the wound $(1=$ not satisfied to $10=$ very satisfied). Scar scale (7.2 \pm 1.3 vs. $3.4 \pm 2.1, \mathrm{P}<0.001)$ and satisfaction with the wound $(7.9 \pm 1.8$ vs. $3.6 \pm 2.0, \mathrm{P}<0.001)$ scores were significantly higher among parents in the LEC than the OEC group.

No patient in either group experienced any critical intraoperative complications, such as hepatic artery injury

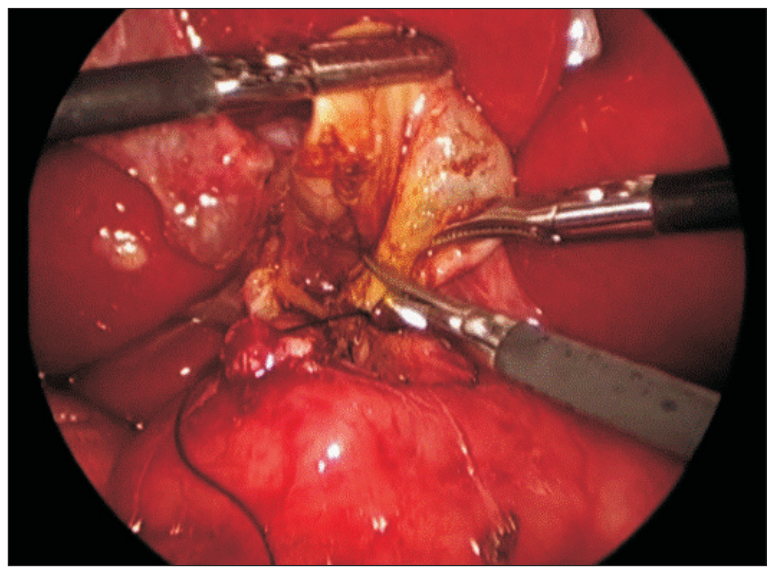

Fig. 2. The distal portion of cyst was cut.

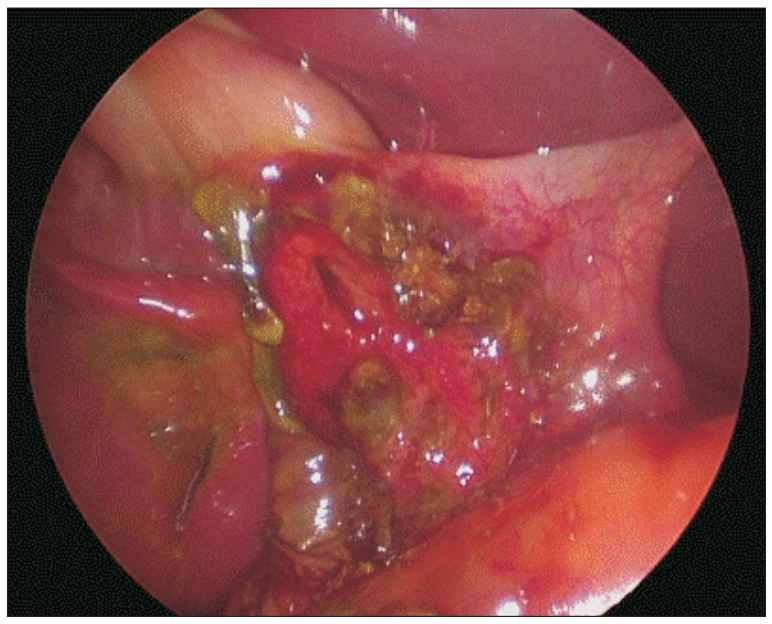

Fig. 3. Ruptured cyst with severe inflammation. 
Table 1. Demographic characteristics of patients included in this study

\begin{tabular}{|c|c|c|c|c|}
\hline Characteristic & OEC $(n=21)$ & $\operatorname{LEC}(n=22)$ & Total $(n=43)$ & P-value \\
\hline Sex, male:female & $4: 17$ & $3: 19$ & $7: 36$ & 0.698 \\
\hline Gestational age (wk) & $39(38-40)$ & $38(37-39)$ & $39(38-39.3)$ & 0.003 \\
\hline Birth weight (g) & $3,420(3,129-3,637)$ & $3,148(2,879-3,314)$ & $3,240(2,955-3,444)$ & 0.229 \\
\hline Age at surgery (PNA, day) & $13(9.5-21)$ & $14(7-22)$ & $13(9-22)$ & 0.759 \\
\hline Weight at surgery (kg) & $3.5(3.27-3.85)$ & $3.35(3.09-3.70)$ & $3.43(3.14-3.70)$ & 0.828 \\
\hline Type of choledochal cyst & & & & 0.515 \\
\hline I & $15(71.4)$ & $18(81.8)$ & $33(76.7)$ & \\
\hline II & $1(4.8)$ & $0(0)$ & $1(2.3)$ & \\
\hline III & $0(0.0)$ & $0(0)$ & $0(0)$ & \\
\hline Iva & $5(23.8)$ & $3(13.6)$ & $8(18.6)$ & \\
\hline $\mathrm{IVb}$ & $0(0)$ & $1(4.6)$ & $1(2.4)$ & \\
\hline Cyst size $(\mathrm{cm})$ & $4.0(2.6-6.0)$ & $3.35(2.5-4.5)$ & $3.80(2.6-5.0)$ & 0.133 \\
\hline Underlying disease & $0(0)$ & $1^{\text {a) }}(4.6)$ & $1(2.4)$ & \\
\hline \multicolumn{5}{|c|}{ Preoperative laboratory parameters } \\
\hline Total bilirubin (mg/dL) & $8.8(6.0-10.8)$ & $9.5(5.2-12.9)$ & $9.1(5.4-11.5)$ & 0.594 \\
\hline Direct bilirubin (mg/dL) & $0.6(0.3-2.9)$ & $0.7(0.6-2.5)$ & $0.7(0.48-2.6)$ & 0.701 \\
\hline AST (IU/L) & $45(35-62.5)$ & $33(27-42.3)$ & $38(28-51)$ & 0.039 \\
\hline ALT (IU/L) & $18(8.50-32)$ & $11.5(6.8-16.0)$ & $13(7-20)$ & 0.007 \\
\hline r-GT (IU/L) & $193.5(89-450.5)$ & $148(95-211)$ & $164(96-256)$ & 0.561 \\
\hline ALP (IU/L) & $193(140-287.5)$ & $165(137-221.5)$ & $180(139-227)$ & 0.589 \\
\hline
\end{tabular}

Values are presented as median (interquartile range) or number (\%).

OEC, open excision of cyst; LEC, laparoscopic excision of cyst; PNA, postnatal age; r-GT, gamma-glutamyltrans-peptidase.

${ }^{a)}$ This patient had congenital anomalies of the heart, including tetralogy of Fallot, patent ductus arteriosus, and patent foramen ovale.

Table 2. Perioperative outcomes

\begin{tabular}{|c|c|c|c|c|}
\hline Variable & $\mathrm{OEC}(\mathrm{n}=21)$ & $\operatorname{LEC}(n=22)$ & Total $(n=43)$ & P-value \\
\hline \multicolumn{5}{|l|}{ Perioperative } \\
\hline Ruptured cyst & $3(14.3)$ & $0(0.0)$ & $3(7.0)$ & 0.234 \\
\hline Pancreatitis & $2(9.5)$ & $2(9.1)$ & $4(9.3)$ & $>0.999$ \\
\hline Transfusion & $2(9.5)$ & $0(0.0)$ & $2(4.7)$ & 0.489 \\
\hline Intraoperative peak $\mathrm{PaCO}_{2}(\mathrm{mmHg})$ & $45.5(42.0-52.5)$ & $48.0(41.0-53.25)$ & $46.3(41.8-53.0)$ & 0.861 \\
\hline Anastomosis size $(\mathrm{mm})^{\mathrm{a})}$ & $5.7 \pm 4.6$ & $6.0 \pm 3.1$ & $5.9 \pm 3.7$ & 0.788 \\
\hline Total operation time (min) & $208.3 \pm 71.0$ & $235.0 \pm 47.2$ & $222.0 \pm 60.8$ & 0.158 \\
\hline Hospital days & $9.9 \pm 5.9$ & $10.8 \pm 5.0$ & $10.5 \pm 5.7$ & 0.588 \\
\hline Initial feeding, POD & $4.2 \pm 1.2$ & $4.4 \pm 2.1$ & $4.3 \pm 1.7$ & 0.742 \\
\hline Full feeding, POD & $6.6 \pm 1.8$ & $7.1 \pm 2.5$ & $6.8 \pm 2.2$ & 0.474 \\
\hline Time to jaundice free (day) & $52.0 \pm 53.1$ & $36.0 \pm 43.4$ & $43.6 \pm 48.3$ & 0.295 \\
\hline \multicolumn{5}{|l|}{ Wound satisfaction } \\
\hline Scar scale & $3.4 \pm 2.1$ & $7.2 \pm 1.3$ & & $<0.001$ \\
\hline Satisfaction score & $3.6 \pm 2.0$ & $7.9 \pm 1.8$ & & $<0.001$ \\
\hline
\end{tabular}

Values are presented as number $(\%)$, median (interquartile range), or mean \pm standard deviation.

POD, postoperative day; OEC, open excision of cyst; LEC, laparoscopic excision of cyst; $\mathrm{PaCO}_{2}$, partial pressure of arterial carbon dioxide.

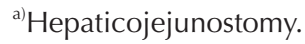

or bile duct injury. Three patients in the OEC group required readmission because of cholangitis and 2 patients had ileus. One of the latter underwent bowel resection because of bowel strangulation by a fibrous band between the jejunojejunostomy site and the midcolon. None of the patients in the LEC group experienced any postoperative complications during follow-up
(Table 3).

\section{DISCUSSION}

Early correction is recommended as soon as CC is diagnosed. In patients with antenatally diagnosed CCs, sludge starts to 
Table 3. Complications in this patient cohort

\begin{tabular}{lllll}
\hline \multicolumn{1}{c}{ Variable } & $\begin{array}{c}\text { OEC } \\
(\mathrm{n}=21)\end{array}$ & $\begin{array}{c}\text { LEC } \\
(\mathrm{n}=22)\end{array}$ & $\begin{array}{c}\text { Total } \\
(\mathrm{n}=43)\end{array}$ & P-value \\
\hline $\begin{array}{l}\text { Intraoperative } \\
\quad \text { Hepatic artery injury }\end{array}$ & $0(0)$ & $0(0)$ & $0(0)$ & \\
$\quad \begin{array}{l}\text { Bile duct injury } \\
\text { Postoperative }\end{array}$ & $0(0)$ & $0(0)$ & $0(0)$ & \\
$\quad$ Wound complication & $0(0)$ & $0(0)$ & $0(0)$ & \\
$\quad \begin{array}{l}\text { Bleeding or } \\
\quad \text { hematoma }\end{array}$ & $0(0)$ & $0(0)$ & $0(0)$ & \\
$\quad \begin{array}{llll}\text { Cholangitis } \\
\text { Ileus }\end{array}$ & $3(14.3)$ & $0(0)$ & $3(7.0)$ & $<0.001$ \\
$\quad$ Hepaticojejunostomy & $0(0)$ & $0(0)$ & $0(0)$ & \\
$\quad$ leakage & & & & \\
$\quad$ Remnant choledocal & $0(0)$ & $0(0)$ & $0(0)$ & \\
$\quad$ cyst & & & & \\
$\quad$ Late pancreatitis & $0(0)$ & $0(0)$ & $0(0)$ & \\
$\begin{array}{l}\text { Readmission } \\
\text { Reoperation }\end{array}$ & $5(23.3)$ & $0(0)$ & $5(11.6)$ & 0.049 \\
\hline
\end{tabular}

Values are presented as number (\%).

OEC, open excision of cyst; LEC, laparoscopic excision of cyst.

form as early as the first 2 weeks of life, with hepatic fibrosis tending to develop from the CC, suggesting that CCs be corrected during these first 2 weeks [13]. To avoid complications such as biliary cirrhosis and cyst rupture, surgery should be performed as soon as possible in patients younger than 1 year [14-16].

Few studies to date have evaluated outcomes of LEC in neonates, because of difficulties performing laparoscopic operations in neonates. These difficulties include a small operative space, risk of injury to vital structures, and poor tolerance to extended periods of pneumoperitoneum [8]. Improvements in surgical techniques and instruments, as well as better understanding of anesthesia in newborns, have increased the number of centers performing LEC $[8,17,18]$. Our center, which includes anesthesiologists specialized in pediatric patients, has accumulated experience performing LEC in children as well as other laparoscopic surgical procedures in neonates. This provided a basis for performing LEC in neonates.

To avoid complications from rapid insufflation of carbon dioxide, the flow rate was set below $3 \mathrm{~L} / \mathrm{min}$ and peak pressure was maintained below $10 \mathrm{mmHg}$, thereby preventing high peak insufflation pressure. Intraoperative peak $\mathrm{PaCO}_{2}$ in the LEC group was safe and did not differ significantly from that in the OEC group (45.5 mmHg vs. $48.0 \mathrm{mmHg}, \mathrm{P}=0.861$ ). Optimal $\mathrm{PaCO}_{2}$ goals for neonates have not been determined, but mild permissive hypercapnia was shown to be safe [19], suggesting pneumoperitoneum can be safely created during laparoscopic surgery in neonates.

To secure visibility of the operative field, we pulled the

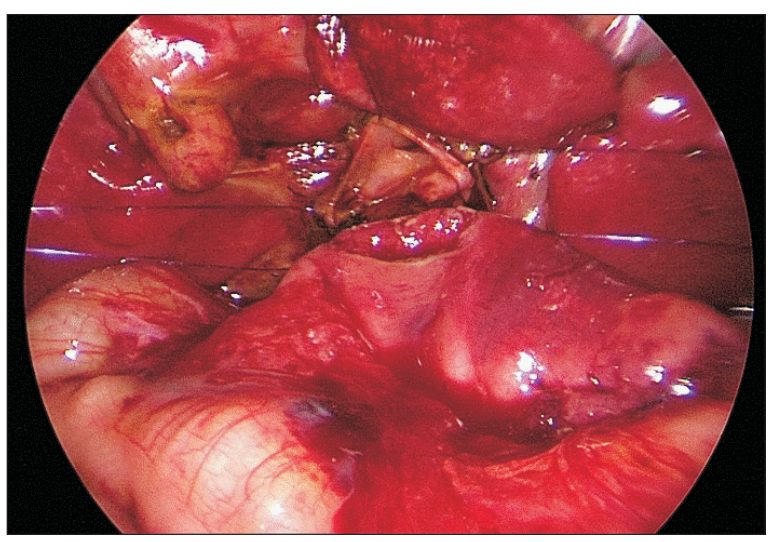

Fig. 4. The shape of an isosceles triangle to provide a better view for anastomosis.

falciform ligament, the gallbladder fundus, and the cystic duct to the abdominal wall and fixed each by ligating with 3-0 prolene sutures through the abdominal wall penetration. Stay sutures were most important for performing stable anastomosis. The anterior part of the hepatic duct margin was hung in the direction of the chest wall, and the lateral part of the hepatic duct margin was sutured to the end of the incision of the jejunum on both sides. In this way, the hepatic duct margin formed the shape of an isosceles triangle (Fig. 4). Among the advantages of this procedure was its ability to provide a better view for anastomosis. Moreover, the posterior side of the hepatic duct was visible, thereby avoiding injury to the hepatic artery. Extension of the hepatic duct enabled suturing at even intervals. These procedures made possible meticulous and safe operations in neonate.

Use of the same procedure for both open and laparoscopic surgery was key to the lack of difference in postoperative complication rates between the 2 groups. There were no critical complications, such as bleeding, anastomosis leakage, remnant CC and late pancreatitis, in both groups. The minimallyinvasive procedure resulted in a significantly lower ileus rate than in the OEC group. Although there was no significant difference in diet, patients in the LEC group were more likely to start early feeding. To minimize vomiting and aspiration, sips were allowed on the third postoperative day, and feeding was started on the fourth day. In addition, LEC provided better cosmetic results and greater satisfaction with the wound than open surgery, with comparable rates of postoperative clinical outcomes. A large multicenter series also reported that the complication rate in children with CCs was significantly lower in those who underwent laparoscopic than open hepaticojejunostomy $(4.2 \%$ vs. $50 \%, \mathrm{P}<0.001)$ [10].

Increased experience has made laparoscopic procedures possible, even in patients with very large sized or ruptured cysts. The largest cyst in our study was $4.6 \mathrm{~cm}$ in diameter. 
This patient underwent LEC without much difficulty. However, laparoscopic procedures always be performed carefully in neonates because it is difficult to identify bile duct variations and there is a risk of hepatic artery injury.

The findings of this study suggest LEC was safe and feasible in neonate. The primary limitation of this study was the small number of patients, making statistical analyses difficult. Prospective randomized studies including a large number of patients are warranted.

In conclusion, despite difficulties performing laparoscopic surgery in neonates, LEC was found to be safe and feasible with acceptable outcomes when intraperitoneal peak pressure was maintained under $10 \mathrm{mmHg}$ and $\mathrm{PaCO}_{2}$ was closely monitored by a pediatric anesthesiologist. Compared with OEC, LEC improved cosmetic outcomes without severe complications. Prospective randomized studies with a large number of patients are needed to better assess the advantages of LEC.

\section{CONFLICTS OF INTEREST}

No potential conflict of interest relevant to this article was reported.

\section{REFERENCES}

1. Miyano T, Yamataka A, Kato Y, Segawa O, Lane G, Takamizawa S, et al. Hepaticoenterostomy after excision of choledochal cyst in children: a 30-year experience with 180 cases. J Pediatr Surg 1996;31:1417-21.

2. Miyano T, Yamataka A, Li L. Congenital biliary dilatation. Semin Pediatr Surg 2000;9:187-95.

3. Ohi R, Yaoita S, Kamiyama T, Ibrahim M, Hayashi Y, Chiba T. Surgical treatment of congenital dilatation of the bile duct with special reference to late complications after total excisional operation. J Pediatr Surg 1990;25:613-7.

4. Farello GA, Cerofolini A, Rebonato M, Bergamaschi G, Ferrari C, Chiappetta A. Congenital choledochal cyst: video-guided laparoscopic treatment. Surg Laparosc Endosc 1995:5:354-8.

5. Li L, Feng W, Jing-Bo F, Qi-Zhi Y, Gang L, Liu-Ming $\mathrm{H}$, et al. Laparoscopic-assisted total cyst excision of choledochal cyst and Roux-en-Y hepatoenterostomy. J Pediatr Surg 2004:39:1663-6.

6. Li L, Liu SL, Hou WY, Cui L, Liu XL, Jun $Z$, et al. Laparoscopic correction of biliary duct stenosis in choledochal cyst. J Pediatr Surg 2008:43:644-6.

7. Lima M, Gargano T, Ruggeri G, Destro F, Maffi M. Laparoscopic treatment of congenital choledochal cyst and hepa- ticojejunostomy with extracorporeal Roux-en-Y anastomosis: technical aspects and early experience with three cases. Pediatr Med Chir 2016;38:125.

8. Diao M, Li L, Cheng W. Laparoscopic versus Open Roux-en-Y hepatojejunostomy for children with choledochal cysts: intermediate-term follow-up results. Surg Endosc 2011;25:1567-73.

9. Liuming H, Hongwu Z, Gang L, Jun J, Wenying H, Wong KK, et al. The effect of laparoscopic excision vs open excision in children with choledochal cyst: a midterm follow-up study. J Pediatr Surg 2011;46:662-5.

10. Qiao G, Li L, Li S, Tang S, Wang B, Xi H, et al. Laparoscopic cyst excision and Roux-Y hepaticojejunostomy for children with choledochal cysts in China: a multicenter study. Surg Endosc 2015;29:140-4.

11. Fujimoto T, Segawa O, Lane GJ, Esaki S, Miyano T. Laparoscopic surgery in newborn infants. Surg Endosc 1999;13: 773-7.

12. Gueugniaud PY, Abisseror M, Moussa M, Godard J, Foussat C, Petit P, et al. The hemodynamic effects of pneumoperitoneum during laparoscopic surgery in healthy infants: assessment by continuous esophageal aortic blood flow echo-Doppler. Anesth Analg 1998;86:2903.
13. O'Neill JA Jr. Choledochal cyst. Curr Probl Surg 1992;29:361-410.

14. Chen CJ. Clinical and operative findings of choledochal cysts in neonates and infants differ from those in older children. Asian J Surg 2003;26:213-7.

15. Liu SL, Li L, Hou WY, Zhang J, Huang LM, Li X, et al. Laparoscopic excision of choledochal cyst and Roux-en-Y hepaticojejunostomy in symptomatic neonates. J Pediatr Surg 2009;44:508-11.

16. Saito T, Terui K, Mitsunaga T, Nakata M, Kuriyama Y, Higashimoto Y, et al. Role of pediatric endoscopic retrograde cholangiopancreatography in an era stressing less-invasive imaging modalities. J Pediatr Gastroenterol Nutr 2014:59:204-9.

17. Lee H, Hirose S, Bratton B, Farmer D. Initial experience with complex laparoscopic biliary surgery in children: biliary atresia and choledochal cyst. J Pediatr Surg 2004:39:804-7.

18. Ure BM, Schier F, Schmidt AI, Nustede R, Petersen C, Jesch NK. Laparoscopic resection of congenital choledochal cyst, choledochojejunostomy, and extraabdominal Roux-en-Y anastomosis. Surg Endosc 2005;19:1055-7.

19. Thome UH, Ambalavanan N. Permissive hypercapnia to decrease lung injury in ventilated preterm neonates. Semin Fetal Neonatal Med 2009;14:21-7. 